\title{
Applications of New Power Line Communication Model for Smart Grids
}

\author{
Derya Betul Unsal ${ }^{*}$, Tankut Yalcinoz ${ }^{2}$ \\ ${ }^{1}$ Department of Electrical and Electronics Engineering, Cumhuriyet University, Sivas, Turkey. \\ 2 Department of Electrical and Electronics Engineering, Mevlana University, Konya, Turkey. \\ * Corresponding author. Tel.: 03462191010; email: dbunsal@cumhuriyet.edu.tr \\ Manuscript submitted December 8, 2014; accepted March 15, 2015. \\ doi: 10.17706/ijcee.2015.7.3.168-178
}

\begin{abstract}
A new grid system structure requirement would be irresistible in the near future. That system should be able to provide remote controls for the development of more efficient, more convenient and more reliable systems. In the smart grids, data transmission and its communication infrastructure in order to better control will be an important function. Therefore, control requirements and communication structures of electrical grids are examined in this paper. The power line communication (PLC) system is discussed for the smart grid system. Then new PLC system model is created, which is used in smart grid communication system, is simulated by Matlab Simulink $\circledR^{\circledR}$. The simulation results have been achieved then tested to applicability of smart grid communication system.
\end{abstract}

Key words: Attenuation, communication, smart grid, power line.

\section{Introduction}

Electricity is generated in huge power plants and transmitted by interconnected high voltage transmission lines in today's electrical grid systems. But there is not a very cost-effective control system on the lines. Because of nonexistence of effective transmission, a possible power cut on high voltage transmission lines can affect other grids negatively. For these reasons, the request for a new network is emerging but it will be used in network communication system will be much more important concept. Traditional grid includes energy centrals which are connected with long transmission lines and they has a connection structure that can be cause blackouts, also if there were any other connected countries, by cut-offs. Smart grid systems improve the reliability of electrical power systems, provide more power quality, reduce time delays, can control resist attacks and etc. A variety of candidate technologies such as fiber optic, wireless and power line communication (PLC) is available for the smart grid communication infrastructure [1], [2].

However, it is important that how this communication will be achieved the most efficient manner.

\section{Smart Grid Communication Technologies and Applications}

As described previously, smart grid is a system which working with power grid and communication technologies on its applications. The best communication methods must be applied all the lines in power line systems to achieve the most effective distribution and transmission systems. Presently, the most important point is designing a communication system architecture that a solution for interconnected systems which can be using at the future smart grid applications [3]. 
Smart grid communication methods generally can be divided into wired and wireless communication methods.

\subsection{Wireless Communication}

Wireless communication provides communication up to places where cannot be reached with cables but it has low data transmission rates. Also it has not a wide range transmission network, generally it is pointed to use in HAN and NAN communication with wireless communication. Wireless communication provides communication up to places where cannot be reached with cables but it has low data transmission rates. Also it has not a wide range transmission network, generally it is pointed to use in HAN and NAN communication with wireless communication.

\subsubsection{Wi-Fi}

Wireless-fidelity (Wi-Fi) is a technology which allows wireless communication of all devices that connected to it. Through the agency of Wi-Fi products, which want to connect a network, connect to LAN with Personal Access Points (PAPs). PAPs connect to wired internet network by a router and transmit receiver data signals to media as RF signals. In addition, PAPs are also used to strengthening received the RF signals from a wireless transmitter to go with increased range. All these connections are determined by IEEE 802.11a, 802.11b, 802.11g and 802.11 standards [4], [5].

\subsubsection{WiMax}

WiMAX is Worldwide Interoperability for Microwave Access technology and a part of 802.16 standard series for Wireless Metropolitan Area Network (WMAN) [5]. According to this standards, it defined the wide operating range of $10-66 \mathrm{GHz}$ for communication infrastructure [6].

\subsubsection{Cellular communication (satellite)}

Cellular communication uses $2 \mathrm{G}$ standards that defined for $1.9 \mathrm{GHz}$ band and have GSM, IS-36 and IS-95 licenses. The 3G and 4G cellular technology operates on $824-894 \mathrm{mhz} / 1900 \mathrm{mhz}$ spectrum range [6]. It used in the latest technology whereas LTE and LTE-A standards [7]. It has features that separate from other wireless communications technologies make it more applicable for smart grid. For example it has high volume capacity that can move huge amount of data on the smart grid applications. And the power grids are already being used it, therefore initial investment cost does not exist, so the data can be transmitted with existing infrastructure. In addition to the cellular communication system has developed infrastructure security [8], [9].

\subsubsection{Bluetooth}

Bluetooth technology operates in the $2.4 \mathrm{GHz}$ ISM frequency band and it is able to transmit voice and data. Bluetooth-enabled devices capable of transferring data at up to $24 \mathrm{mbps}$ are the effective distance is about 10 to 100 meters. Bluetooth installed on small, high-performance integrated radio transceiver units. Each of these units, with addresses that are derived from the IEEE 802.11 standards and it is capable of high data transmission with low power consumption. [10] This technology is suitable for the use of the Smart Grid LAN networks.

\subsubsection{ZigBee}

ZigBee takes its name from the zigzag area of the complex structure and based on the IEEE 802.15.4 standard. The purpose of that standard helps to creation of personal wireless networks which has low infrastructure cost, slow transfer rate and low power consumption [11]. Although ZigBee has advantages like long battery life, requested network building, it has the disadvantage of not able to provide data flow in the larger sizes as Bluetooth and Wi-Fi. It is suitable for HAN areas accepted of the smart grid [12].

\subsection{Wired Communications}


Wired communication infrastructure can be divided into Fiber optics and PLC's. These methods' challenges and benefits investigated carefully. As described previously, Smart Grid networks can be included HANs, BANs, LANs, wired and wireless networks that connect power generation systems to end consumers in order to support a wide range of communication and control applications including demand response and distribution automation [13].

\subsubsection{Fiber optics}

Substations and electricity companies which connected on the power system can reach high-speed level communicate on broadband with each other by Fiber optics technology. Despite the fact that it has a high initial setup cost disadvantage, is not affected by electromagnetic fields and wired communication has all the advantages of security through privileges are preferred by smart grid applications. It is not possible to steal information because from the privacy and security are very good about fiber cable [14].

\subsubsection{Power line communication (PLC)}

Power line communication systems include all the advantages of fiber-optic cable and fast data communication along with the security of wireless communication methods. Communication is meaning that not just only internet connection, it means also every device connected to the network on power line. PLC can also control active and passivity of distribution lines. This is essential especially for substations located in countrified areas where there isn't any communication infrastructure. PLC technology usually uses for data communication medium and low voltage power lines [7]. PLC uses the existing wiring, so it is suitable for use in HAN and NAN [15], [16]. PLC technology provides high data transmission on short field. For example, in a building as LAN network, bits per second data transmission rate can rise up to million bits per second with PLC. However, the data transmission must be made with the appropriate of PLC technology. Different technologies of PLC use different frequency band scales and provide different data transmission rates. Data communication technologies which used in PLC can be divided into BPLC and NBPLC.

- Broadband Power Line Communication (BPLC):

BPLC uses a standard for high-speed, which has over 100 Mbps speed at the physical layer, communication assets by electric power lines. This standard [17] uses transmission frequencies below 100 MHz. It is applicable for all classes of BPLC devices, including BPLC devices used for the first-mile/last-mile connection, which under the $1500 \mathrm{~m}$ to the premise, to broadband services as well as BPLC devices used in buildings for LANs, smart grid applications and other data distribution which has less than $100 \mathrm{~m}$ distance between devices [17]. A wide area communication is available from home automation to access the internet through the power lines.

- Narrowband Power Line Communication (NBPLC):

NBPLC technology uses standard specifies communications for low-frequency, which has less than 500 $\mathrm{kHz}$ frequency value, assets through alternating current and direct current electric power lines. This standard [18] supports indoor and outdoor communications with low voltage and medium voltage power lines through incorporating transformers in both long distance rural and urban applications with transmission frequencies less than $500 \mathrm{kHz}$. Application requirements and network conditions effected to data rates will be measured to $500 \mathrm{~kb} / \mathrm{s}$ [18]. This technology can be used with grid automation applications and within HAN communications scenarios. NBPLC is used with frequency spectrum from 9 to $140 \mathrm{kHz}$ for PLC applications [19]. NBPLC technology seems to be cost-effective, has more security and reliability, can appropriate to meet the bandwidth requirements of especially in large scale smart grid communication applications [20]. In the below diagram Fig. 1 seems a BPLC and NBPLC technologies use in different length of transmission lines.

When decide the most suitable communication method for smart grids, which has the lowest cost, the largest gain, error detection ability of loss and leakage rates, Table 1 summarizes the comparison of wired 
and wireless communication.

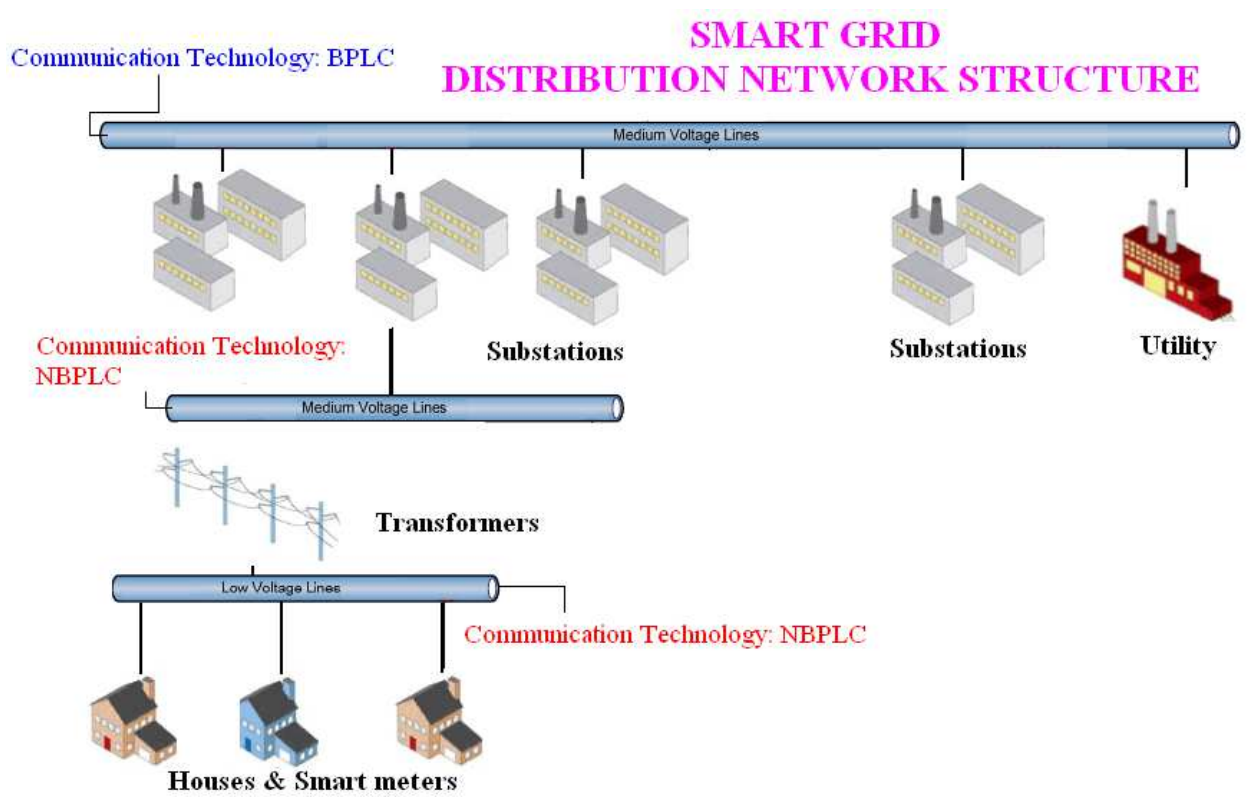

Fig. 1. Smart grid distribution network architecture.

Table 1. Comparison of wired and wireless communication [21], [22]

\begin{tabular}{|l|l|l|}
\hline Technology & Advantages & Disadvantages \\
\hline Wired-PLC & $\begin{array}{l}\text { Cost-Effective } \\
\text { Available Infrastructure } \\
\text { Extensive Coverage } \\
\text { High capacity } \\
\text { Security }\end{array}$ & $\begin{array}{l}\text { Signal attenuation } \\
\text { High noise }\end{array}$ \\
\hline Wired-Optical Fiber & $\begin{array}{l}\text { Stable characteristics } \\
\text { High capacity }\end{array}$ & Cost \\
\hline \multirow{2}{*}{ Wireless } & Rapid installation & $\begin{array}{l}\text { Limited coverage } \\
\text { Cost } \\
\text { Security } \\
\text { Capacity } \\
\text { Long delay }\end{array}$ \\
\hline
\end{tabular}

PLC is the most cost-effective wired communication in this dissertations result, and Wireless Communication have the best applicable network structures to provide deployment of smart grid communication, they are cost-effective technologies which minimize additional investment in infrastructure [1]. But wireless communication has more disadvantages than wired communications as cost, security, capacity, etc. Therefore the wired communication is more effective for smart grid applications. In the other applications, PLC is at the introduction stage or has just started growing. It looks that it will be continue to growing. It seems that power lines reach everywhere from countrified areas to metropolitans but it suffers working electrical machines noise and signal attenuations and transformers because of their high inductances act as low pass filters and may block high frequencies. But these problems can be solve, BPLC couplers are required to be attached at the transformers to allow high frequencies to pass through them, also attenuation is increased by channel characteristics [23]. So, if minimized attenuation and noise problems of PLC, the most appropriate method can be used as is evident.

\section{PLC System Modeling for Smart Grid Communication Applications}

PLC technology usually uses for data communication of medium and low voltage power lines. The 
communication signal must be delivered with maximum efficiency on PLC applications. Otherwise, data losses in the transmission can be at high rates. In spite of being cost effective solutions for smart grid applications, PLC environments have great challenges as attenuation (transmission or path loss) to reliability and performance of communication systems [1].

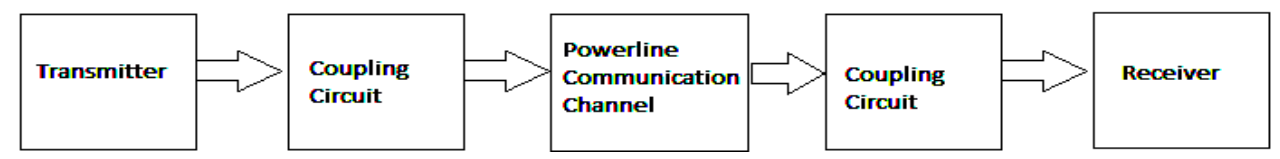

Fig. 2. Power line communication general block diagram.

Fig. 2 shows the general architecture of the PLC system. Tx model represents a block diagram for the generation signal. Rx model represents a block diagram for the receiving signal. The coupling circuit is used a circuit model with coupling transformers.

Tx Model: As shown from Fig. 3, the sinusoidal generator is added in series to an inductor L need to suit the generator output with the coupling network. Besides, the secondary transformer is added in series to a capacitor $\mathrm{C}$ to eliminate the residual $60 \mathrm{~Hz}$ voltage [24].

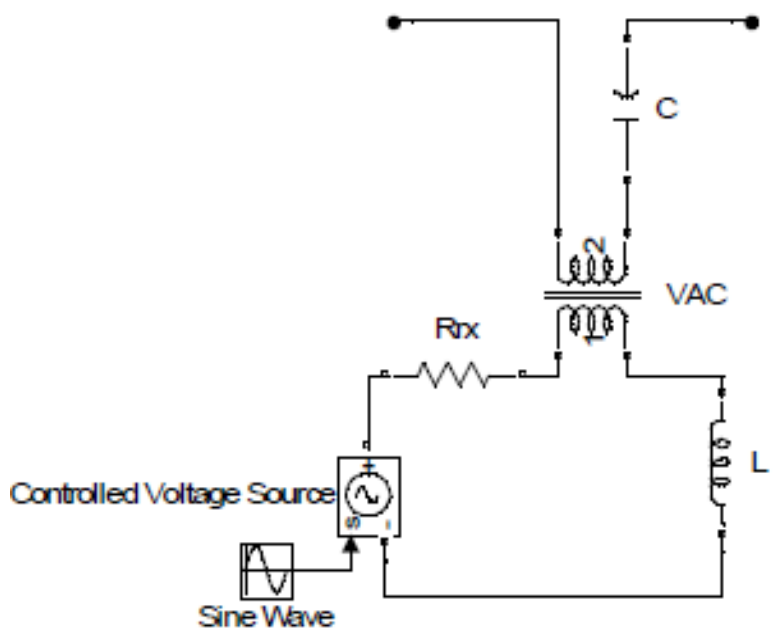

Fig. 3. Tx model [24].

Rx Model: As shown from Fig. 4. The signal is picked up by the power cables through the coupling circuit, in load side, and transferred at the receiver through an isolation transformer [24].

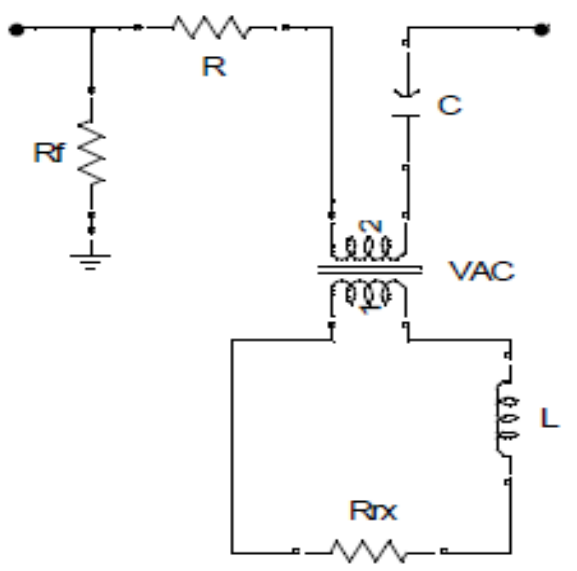

Fig. 4. Rx model [24]. 
Coupling Circuit (CC): Fig. 5 shows a coupling filter circuit on the power line. Tx and Rx circuits are connected by the coupling circuit on a distributed line. It isolate the connected device from the power line between transmitter and receiver. It has capacitor and the self-inductance of the transformer [25]. CENELEC standard is used for determining coupling filter parameters, and the parameters only allow frequencies between $3 \mathrm{kHz}$ and $148.5 \mathrm{kHz}$ [26].

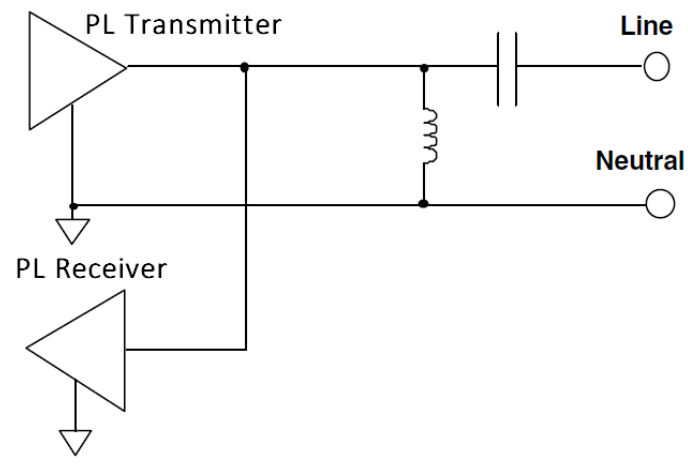

Fig. 5. The coupling filter circuit on the power line [25].

CENELEC A and B standards used for filters in this study, so it is operated on frequencies in the range, the CC filters parameters have been implemented on Simulink [26], [27].

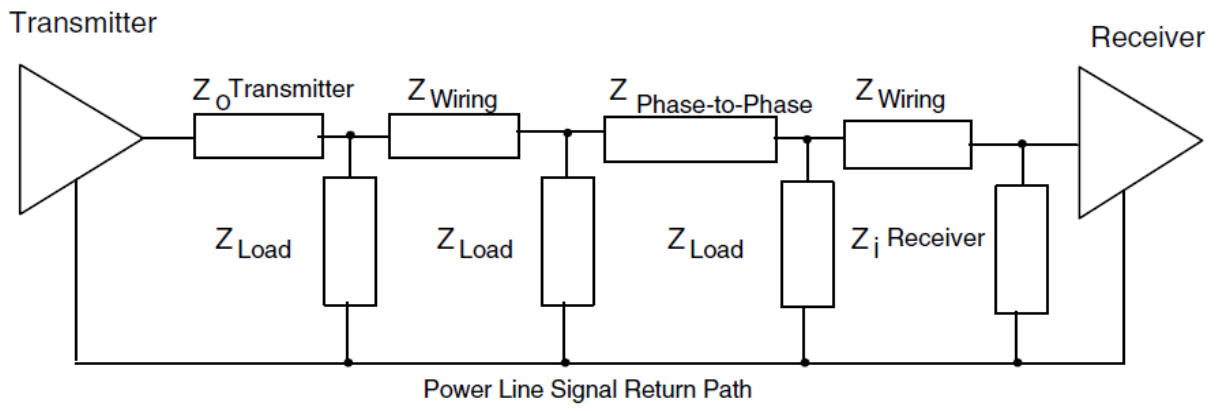

Fig. 6. Power mains attenuation model [25].

Attenuation: Whereas attenuation is technically defined as the ratio of power levels, it is referred to in this study as the ratio of the transmitted signal voltage and the received signal voltage. A voltage ratio is more convenient to measure because power measurements require knowledge of the circuit impedance which varies with both location and time. Fig. 6 illustrates an attenuation model.

The attenuation is defined in $\mathrm{dB}$ as $20 \log _{10}\left(\mathrm{~V}_{\text {transmit }} / \mathrm{V}_{\text {receive }}\right)$. The attenuation has been measured using with MV and LV parameters [24], [28] transmitting a sine wave signal with constant amplitude at the substation. Sine wave is a signal that starts at a given frequency and continuously increase (or decrease) the frequency until it reaches the end frequency. When the sine wave arrives at the receiver it has been affected by the channel and has been attenuated. Because the attenuation varies in frequency, different parts of the sine wave are attenuated differently and the received signal is a measure of the frequency response of the channel [28]-[34]. The amplitude of the transmitted signal has been set to $10 \mathrm{~V}$ (into $50 \mathrm{Ohm}$ ) and the range of the sine wave has been tuned to the frequency band of interest.

\section{PLC System Models with Simulation Results and Discussion}

Different PLC system models are created and examined with transmission behaviors of the test systems. Fig. 2 shows the block diagram of models which created to examine the behavior of the PLC system. 


\subsection{Case Study 1}

The PLC system model 1, as shown in Fig. 7, given in reference [30] is built to examine the behavior of loaded lines characteristics. Fixed-length line measurements results are taken through the transmission lines. LV transmission parameters are applied to the system [17], the input and output voltage ratio are taken as graphically. The long line transmission parameters [26], [28], [31], [33], [35]-[37] are applied on this model.

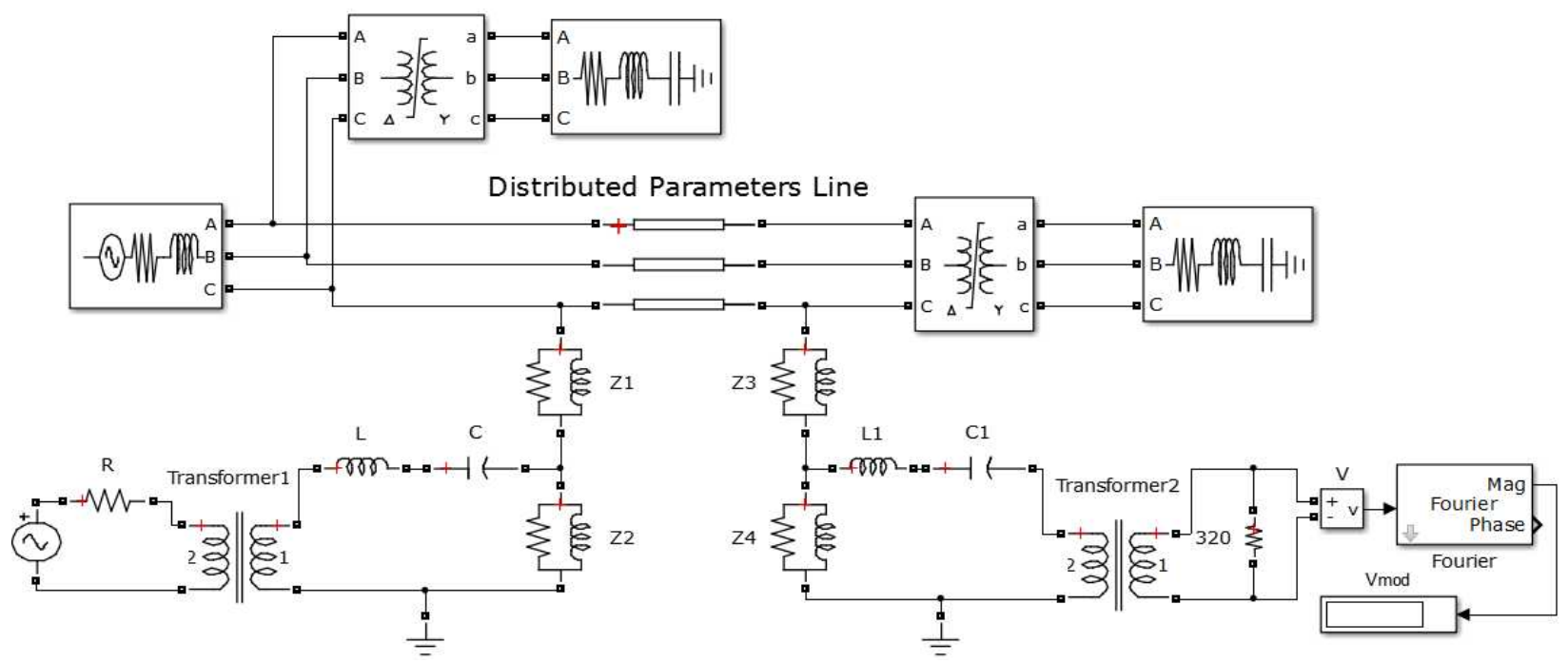

Fig. 7. PLC system model 1 [30].

The results of PLC system model 1 are shown attenuation values of the transmitting signal in the range of 5 to $150 \mathrm{kHz}$ with a magnitude of $10 \mathrm{~V}$ (into $50 \mathrm{ohm}$ ) as shown in Fig. 8. The signal attenuation increases with frequency and above $25 \mathrm{kHz}$. The rate of the output voltage is reduced with increasing load, so these results show that an increasing load is caused higher attenuation.

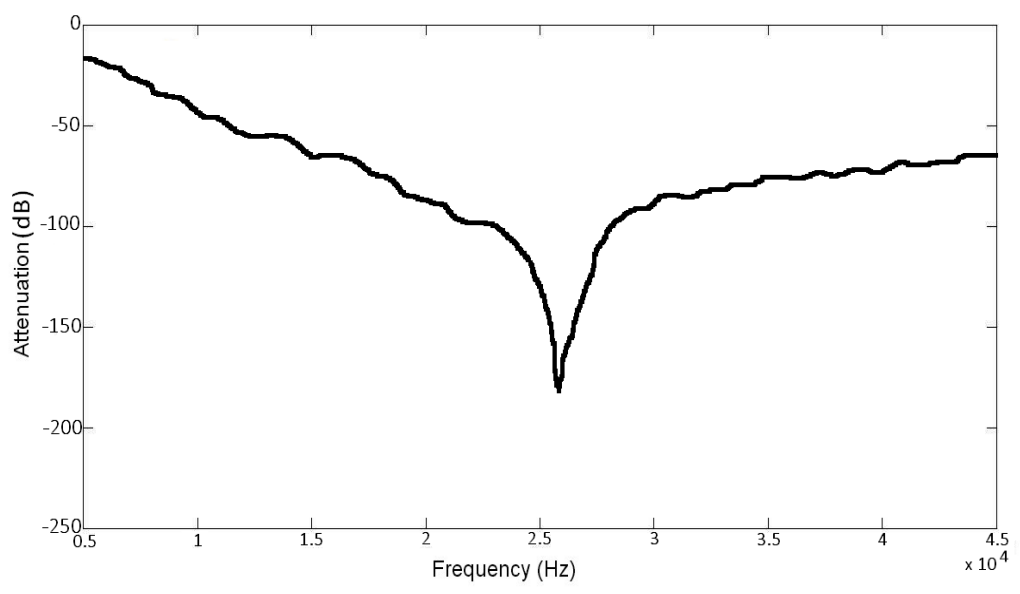

Fig. 8. Attenuation values on PLC system model 1.

\subsection{Case Study 2}

The new power line communication system has been formed in Fig. 9. When designing this model some reference works [24], [29], and [30] has been used. The model of the PLC system consists of the coupling filter, Tx and Rx models. The overhead medium voltage and the low voltage transmission lines are tested 
with a fixed length of the line. Then, the simulation results of the PLC system are obtained for different line lengths in MV transmitted-MV received communication, LV transmitted-LV received communication. The line transmission parameters taken from IEEE and CENELEC standards [17], [26], and [31] are used in the model. This model takes us better transmission results than the others.

The losses that caused by overload is minimized in this model and the most similar model like actual transmission line has been designed.

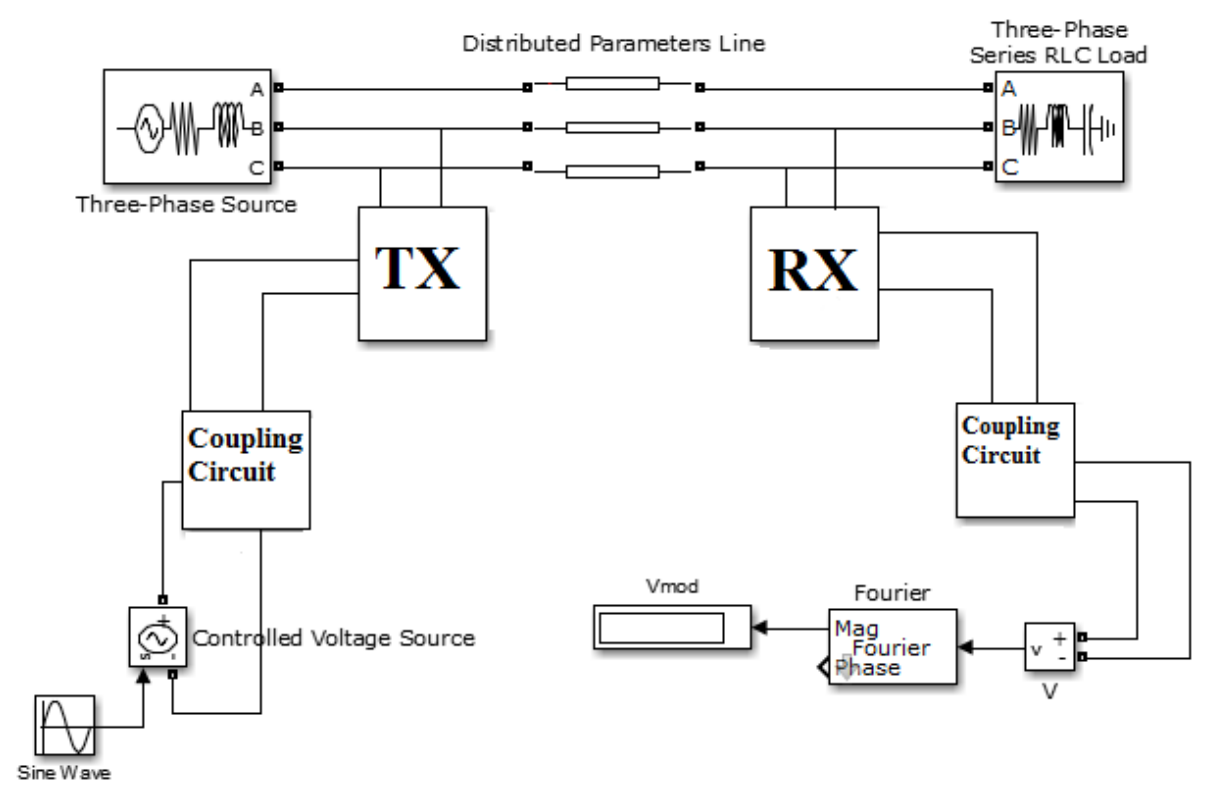

Fig. 9. PLC system model 2.

\subsubsection{MV application results of PLC system model 2}

CENELEC B standards [26] are used for the filter and IEEE parameters [17], [31] are applied on Tx and Rx. The distributed cable line values are taken from [17], [32] in this model. The simulations are performed in frequency ranges of $60-100 \mathrm{kHz}$.

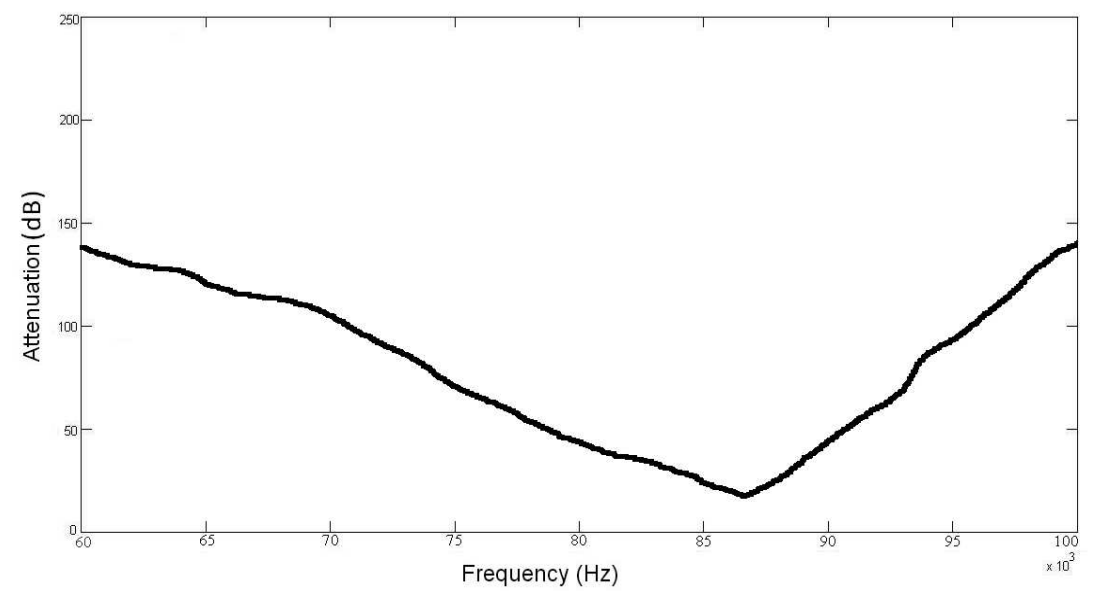

Fig. 10. Attenuation values of PLC transmission system model 2 on MV.

Fig. 10 shows attenuation values of PLC transmission system. According to results, a minimum attenuation value of $18.87 \mathrm{~dB}$ at the frequency of $87 \mathrm{kHz}$ is obtained and it is lower than $50 \mathrm{~dB}$ in the frequency range of $87 \mathrm{kHz} \pm 5 \mathrm{kHz}$. 


\subsubsection{LV application results of PLC system model 2}

According to the same model, CENELEC B standards [26] are used for the filter, LV parameters [17], [24], and [31] are taken for Tx and Rx, also distributed line cable variables are used from references [24], [32] to applied LV transmission.

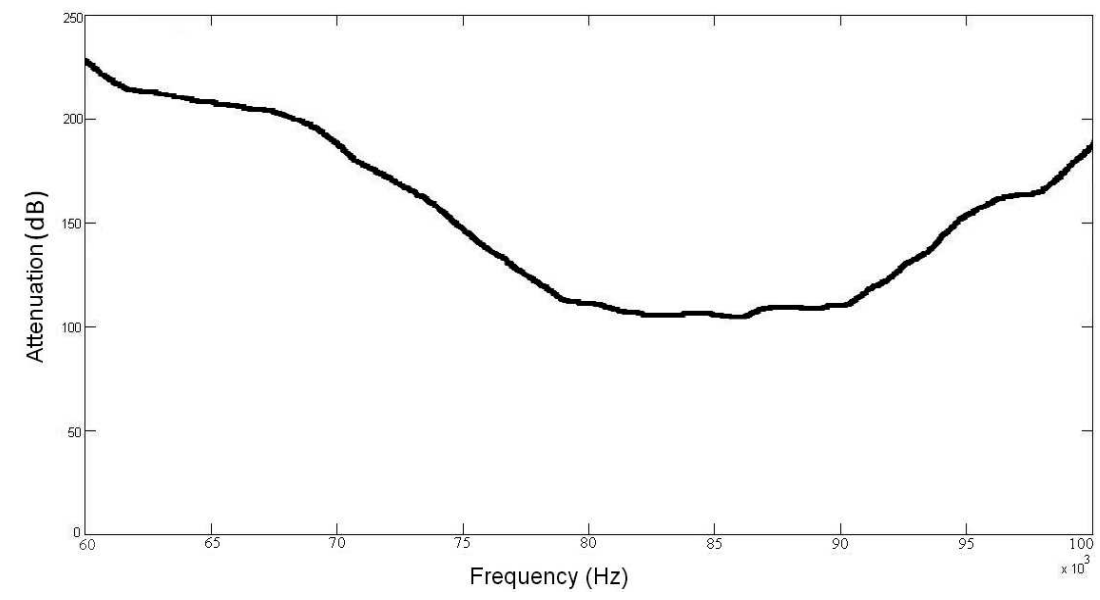

Fig. 11. Attenuation values of PLC transmission system model 2 on LV.

Fig. 11 shows attenuation values of PLC transmission system on LV. The results show a minimum attenuation value of $116.37 \mathrm{~dB}$ at the frequency of $87.81 \mathrm{kHz}$ and it is lower than $100 \mathrm{~dB}$ in the frequency range of $87 \mathrm{kHz} \pm 5 \mathrm{kHz}$. It is clear that, $\mathrm{MV}$ transmissions are observed better results than $\mathrm{LV}$ transmission on this model.

\section{Conclusion}

The effective communication method is an essential requirement for developing the smart grid. There is currently an ongoing debate surrounding what would be the best communication technology for smart grid applications. In this paper, smart grid communication technologies and its requirements are considered and the power line communication system is discussed in detail for the smart grid system. To examine the behaviors of PLC for smart grid applications, different PLC system models are simulated and investigated causes attenuation factors which influence the calculations such as different line lengths or output voltage amplitudes. The overhead medium voltage and the low voltage transmission lines are tested with a fixed length of the line. Then the simulation results of the PLC system are obtained for different line lengths in MV transmitted - MV received communication, LV transmitted - LV received communication. According to simulation results, the comparisons of the medium and low voltage transmitting on different PLC models, the PLC model 2 on MV transmission line has best attenuation values for the power line communication applications.

\section{Acknowledgment}

The author Derya Betül ÜNSAL wish to thank The Scientific and Technological Research Council of Turkey (TUBITAK) for supporting her study.

\section{References}

[1] Guzelgoz, S. (2011). Characterizing wireless and power line communication channels with applications to smart grid networks. PhD Dissertation, University of South Florida.

[2] Shawkat, A. B. M. (2013). Smart Grids: Opportunities, Developments, and Trends. IEEE. 
[3] Sood, V. K., Fischer, D., Eklund, J. M., \& Brown, T. (2009). Developing a communication infrastructure for the Smart Grid. Proceedings of IEEE Electrical Power \& Energy Conference (pp. 1-7).

[4] IEEE Standard 802.16. (2009). IEEE Standard for Local and Metropolitan Area Networks.

[5] IEEE Standard 802.20. (2008). IEEE Standard for Local and Metropolitan Area Networks.

[6] International Telecommunications Union publications. from http://www.itu.int

[7] Ma, R., Chen, H., Huang, R., \& Meng, W. (2013). Smart grid communication: Its challenges and opportunities. IEEE Transactions on Smart Grid, 4(1), 36-46.

[8] Güngör, V. C., Sahin, D., Kocak, T., Ergüt, S., Buccella, C., Cecati, C., \& Hancke, G. P. (November 2011). Smart grid technologies: Communication technologies and standards. IEEE Transactions on Industrial Informatics, 7(4), 529-539.

[9] Gungor, V. C., Sahin, D., Kocak, T., \& Ergut, S. (2013). A Survey on smart grid potential applications and communication requirements. IEEE Transactions on Industrial Informatics, 9(1).

[10] IEEE Standard 802.11. (2012). IEEE Standard for Wireless LANs.

[11] ZigBee homepage. from http://www.zigbee.org

[12] Usman, A., \& Shami, S. (2012). Evolution of Communication Technologies in Smart Grids, Renewable and Sustainable Energy Reviews. Elsevier.

[13] IEEE. (2010). Proceedings of IEEE International Conference on Smart Grid Communications.

[14] Tohumoğlu, Y. (1998). Haberleşmede kullanilan optik Fiber kablolarin ek ve ölçüm metodlari. MS thesis, Niğde University.

[15] Ginot, N., Mannah, M. A., Batard, C., \& Machmoum, M. (2010). Application of power line communication for data transmission over PWM network. IEEE Transactions Smart Grid, 1(2), 178-185.

[16] Bumiller, G., Lampe, L., \& Hrasnica, H. (2010). Power line communication networks for large-scale control and automation systems. IEEE Communications Magazine, 48(8), 106-113.

[17] IEEE Standard 1901 ${ }^{\mathrm{Tm}}$. (2010). IEEE Standard for Broadband over Power Line Networks: Medium Access Control and Physical Layer Specifications.

[18] Korki, M., Hosseinzadeh, N., Vu, H. L., Moazzeni, T., \& Foh, C. H. (2011). A channel model for power line communication in the smart grid. Proceedings of Power Systems Conference and Exposition (pp. 1-7).

[19] NIST Framework and Roadmap for Smart Grid Interoperability Standards. Release 1.0.

[20] Galli, S., Scaglione, A., \& Wang, Z. (Jun. 2011). For the grid and through the grid: The role of power line communications in the smart grid. Proceedings of IEEE, 99(6), 998-1027.

[21] Gungor, V. C., \& Lambert, F. C. (2006). A survey on communication networks for electric system automation. Computer Networks, 50, 877-897.

[22] Haidine, A., Adebisi, B., Treytl, A., Pille, H., Honary, B., \& Portnoy, A. (Apr. 2011). High speed narrowband PLC in smart grid landscape state of the art. Proceedings of IEEE ISPLC. Italy.

[23] Aalamifar, F. (2012). Viability of powerline communication for smart grid realization. MS thesis, Queen's University.

[24] Cataliotti, A., \& Tinè, G. (2009). On the model of MV power line communication system in the case of line to line transmission. Proceedings of XIX IMEKO World Congress Fundamental and Applied Metrology. Lisbon, Portugal.

[25] Echelon Corporation. LonWorks PLT-30 A-Band Power Line Transceiver Module, User's Guide. Version 1.3.

[26] EN 50065-1: 1991 Signaling on low-voltage electrical installations in the frequency range 3 to 148.5 $\mathrm{kHz}$-Part 1: General requirements, frequency bands and electromagnetic disturbances; Amendment A1:1992 to EN 50065-1:1991; Amendment A2:1995 to EN 50065-1:1991; Amendment A3:1996 to EN 50065-1:1991. 
[27] Parikh, P. P., Kanabar, M. G., \& Sidhu, T. S. (July 2010). Opportunities and challenges of wireless communication technologies for smart grid applications. Proceedings of IEEE Power and Energy Society General Meeting (pp. 1-7).

[28] Cataliotti, A., Daidone, A., Sanacore, G., Tinè, G. (September 2007). Characterization of medium voltage cables for power lines communications. Proceedings of 15th IMEKO TC4-International Symposium on Advanced of Measurement Science. Iasi, Romania.

[29] Zimmermann, M., \& Dostert, K. (April 2002). A multipath model for the power line channel. IEEE Transactions on Communications, 50(4), 553-559.

[30] Cataliotti, A., Cara, D. D., \& Tinè, G. (2010). Model of line to shield power line communication system on a medium voltage network. Proceedings of IEEE Instrumentation and Measurement Technology Conference.

[31] IEEE Standards 643"М . (June 8, 2005). IEEE Guide for Power Line Carrier Applications.

[32] RG7H1R Elektrotek medium voltage cables. From http://www.elettrotekkabel.com

[33] Cataliotti, A., Daidone, A., \& Tinè, G. (October 2008). Power line communications in medium voltage system: Characterization of MV cables. IEEE Transactions on Power Delivery, 23(4).

[34] Fröroth, I. (1999). More than power down the line. MSc Thesis, Royal Institute of Technology, Stockholm, Sweden.

[35] ANSI. (2006). Control Network Power line (PL) Channel Specification. ANSI/CEA 709.2 - A - 2000.

[36] Xu, C., Zhou, L., Zhou, J. Y., \& Boggs, S. (Nov.-Dec. 2005). High frequency properties of shielded power cable - Part 1: Overview of mechanism. IEEE Electrical Insulation Magazine, 21(6), 24-28.

[37] Cataliotti, A., Daidone, A., \& Tinè, G. (January 2009). A medium voltage cable model for power line communication. IEEE Transactions on Power Delivery, 24(1), 129-135.

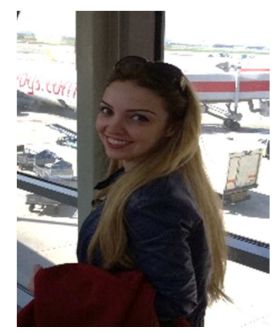

Derya Betül Ünsal received her B.Sc. degree in electrical and electronics engineering in 2011 from Cumhuriyet University. She obtained her M.Sc. degree in electrical and computer engineering with research in the area of smart grids communication from Meliksah University, Kayseri, Turkey in 2014. Currently she is pursuing her PhD degree at Meliksah University. She is a research assistant at Cumhuriyet University. Her areas of research interest are smart grids communication, wireless communication, power line communication, satellite technology. She is a student member of IEEE since 2010.

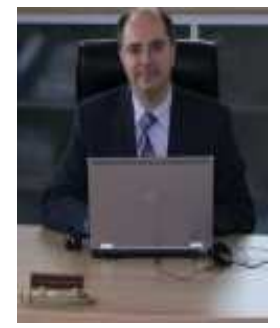

Tankut Yalçinöz received the B.Sc. degree in electrical and electronic engineering from Karadeniz Technical University, Turkey in 1990 and the M.Sc. degree from Cukurova University, Turkey in 1993. In 1998 he obtained a Ph.D. degree from Imperial College, London University in London. He is a professor at Mevlana University, Turkey. He is the author or coauthor of more than 100 published papers, including 48 articles in refereed journals. His main interests are in power system planning and operation, computational intelligence, renewable energy, flexible ac transmission systems, power electronics and fuel cells. He is a senior member of the Institute of Electrical and Electronics Engineers (IEEE). 